\title{
Conducting polymer hydrogel materials for high-performance flexible solid-state supercapacitors
}

\author{
Kai Wang, Xiong Zhang, Xianzhong Sun and Yanwei Ma*
}

\begin{abstract}
Different from solid electrodes, conducting polymer hydrogel electrodes swollen with water and ions, can reach contact with the electrolyte solution at the molecular level, which will result in more efficient electrochemical process of supercapacitors. Besides, the inherent soft nature of hydrogel material offers the electrode superior flexibility, which benefits to gain high flexibility for devices. Here, this perspective briefly introduces the current research progress in the field of conducting polymer hydrogel electrodes-based flexible solid-state supercapacitor and gives an outlook on the future trend of research.
\end{abstract}

Keywords: Conducting polymer hydrogels, flexible supercapacitor, soft electrode, polyaniline, integrated device

Nowadays, flexible energy storage devices (batteries and supercapacitors) have received widely attentions as the rapid progress of flexible and wearable electronics $[1,2]$. Flexible solid-state supercapacitors represent a new type of supercapacitor that can work under consecutive bending, stretching and even twisting states [3-5]. However, it remains a large challenge to obtain a flexible solid-state supercapacitor with high electrochemical performance and superior flexibility. The performance of a flexible supercapacitor mainly depends on the properties of electrode materials and the device configuration [6]. The electrode materials of supercapacitor include various nanocarbons, conducting polymers and transition metal oxides [7-12]. Conducting polymer electrodes possess several merits, such as high electric conductivity, large capacitance, inherent flexibility and easy to processing [13-18]. However, the separations of the molecular chains in conducting polymers are generally small relative to the double layer thickness, which results in low capacitance [19]. Besides, the low ionic mobility in the polymer matrix reduces the maximum power density of such electrodes [20].
On the other hand, the current device configuration of the flexible solid-state supercapacitor somewhat hinders the device to gain better performance. Generally, a flexible solid-state supercapacitor consists of a "sandwich" laminated structure, including two flexible solid electrodes and the middle gel polymer electrolyte layer [21]. In terms of this structure, the solid electrode materials hardly make a sufficient contact with the gel polymer electrolyte that possesses large viscosity and poor diffusion, which results in low capacitance and sluggish the electrochemical response.

Hydrogel is made up of solid three-dimensional polymeric networks and large amounts of water medium [22]. Generally, the hydrogels are employed as solid-state electrolyte of supercapacitors, such as polyvinyl alcohol (PVA) gel polymer electrolyte. Recently, conducting polymer hydrogels were used as electrode materials for supercapacitors instead of traditional solid electrodes [19,23-25]. This "aqua material electrode" is conducting polymer matrix swollen with water and ions, which hence produce an ideal interface between the electrode materials and the electrolyte solution. In other words, the hydrogel electrode materials can maintain contact with the electrolyte solution at the molecular level, resulting in an extremely high effective surface area of electrode in a solid-state supercapacitor device. Besides, the inherent soft nature of hydrogel material offers the electrode superior flexibility, which benefits the preparation of flexible devices.

Here, this perspective briefly introduces the current research progress in the field of conducting polymer hydrogel electrodes-based flexible solid-state supercapacitor.

The preparation and electrochemical performance of conducting polymer hydrogels

MacDiarmid et al. [26] firstly observed that sufficiently

Institute of Electrical Engineering, Chinese Academy of Sciences, Beijing 100190, China

${ }^{*}$ Corresponding author (email: ywma@mail.iee.ac.cn) 
concentrated polyaniline (PANI) solutions tend to gelatinize with time. Since then, the preparation of conducting polymer hydrogels has been intensively investigated in the past decade, involving cross-linking with chemical bonds or physical nodes (crystallites or aggregates) [27-31]. Besides, conducting polymer hydrogels can also be prepared by incorporating of conducting polymers with the host hydrogel matrix [32,33].

The application on supercapacitor of conducting polymer hydrogels was pioneered by Inganas' group, in which the hydrogel of poly(3,4-ethylene-dioxythiophene)poly(styrene sulfonate) (PEDOT-PSS) was produced by an ionically cross-linking process [19]. A PEDOT-PSS electrode was obtained by casting its dispersion onto a gold foil electrode, and then followed by an equilibrating process with $\mathrm{MgSO}_{4}$ solution for ionic crosslinking. However, the mechanical strength of the material is poor and the capacitance is unsatisfied due to the high molecular mass of the PEDOT-PSS. To overcome the capacitance issue, polypyrrole ( $\mathrm{PPy}$, another conducting polymer) was electrochemically deposited on the PEDOT-PSS electrode. The PEDOT-PSS-PPy composite hydrogel possesses less water content and superior mechanical strength that can form a free standing film. Cyclic voltammetry (CV) tests of such electrodes in two-electrode symmetrical supercapacitor were carried out in an aqueous electrolyte (Fig. 1a), illustrating a rectangular shape even at extremely high scan rates, which demonstrates that the high rate performance can be achieved in these electrodes. Besides, both the PEDOT-PSS and PEDOT-PSS-PPy electrodes exhibit the large power densities of over $10 \mathrm{~kW} \mathrm{~kg}^{-1}$ without a significant decrease in the energy (Fig. 1b). The PEDOT-PSS-PPy hydrogel electrode has a maximum energy density of $10 \mathrm{~W} \mathrm{~h} \mathrm{Kg}{ }^{-1}$ that is larger compared to the PEDOT-PSS hydrogel electrode because PPy can store a higher amount of charge per unit weight. Furthermore, incorporating with PPy also reinforces the conducting network of the swollen polymer which also contributes to the high energy density. PPy composite with $95 \%$ water content (known as w-PEDOT-PSS-PPy) shows similar mechanical and electrochemical properties to that of PEDOT-PSS-PPy, and the former even exhibits a better power capability. However, the energy densities of pure PPy-coated electrodes and the un-swollen PEDOT-PSS-PPy electrodes show a sharply decrease as the power increasing. For pure PPy electrodes, only one fifth of energy is obtained when the power density reaches $3 \mathrm{~kW} \mathrm{Kg}{ }^{-1}$, whereas no obvious loss in energy density for PEDOT-PSS-PPy electrodes. Moreover, the swollen electrodes can deliver the energy at power densities more than 25 times higher than that of the un-swollen electrodes, which highlights the merits of the swollen hydrogel materials.

Recently, Pan et al. [23] prepared conducting PANI hydrogels by using phytic acid as gelator and dopants. The gelation mechanism of PANI hydrogel is schemed in Fig. 1c. Phytic acid as dopants can react with PANI molecular by protonating the amino-groups on PANI. The cross-linking occurs due to each phytic acid molecule can interact with more than one PANI chains, resulting in the formation of a hydrogel network. The ionic conductivity of the PANI hydrogel reaches $0.11 \mathrm{~S} \mathrm{~cm}^{-1}$ (293K), which shows a new record conductivity among conducting polymer hydrogels reported to date. The surface morphology of the dehydrated PANI hydrogel shows foam-like porous nanostructures which are constructed with PANI nanofibers with uniform diameters of 60-100 nm, as shown as in Figs 1d and e. This three-dimensional (3D) interconnected porous structure is more effective than wires and particles for supercapacitor applications, which shows high performance for electrochemical capacitors. In a three-electrode testing, the as-prepared PANI hydrogel was loaded on carbon fiber cloth as working electrode with a Pt foil counter electrode and saturated calomel electrode (SCE) reference electrode. The CV curves of PANI hydrogel electrode show no deviation even at high scan rates, demonstrating the fast rate-capability of this hydrogel (Fig. 1f). The electrochemical impedance spectroscopy (EIS) confirms the fast electrochemical process. The electrical resistance is quite small (around $3.2 \Omega$ ) in a $1 \mathrm{~mol} \mathrm{~L}^{-1} \mathrm{H}_{2} \mathrm{SO}_{4}$ electrolyte with a large PANI mass amount $\left(2 \mathrm{mg} \mathrm{cm}^{-2}\right.$ in dehydrated form). The vertical profile of the EIS curve at low frequencies exhibits an ideal capacitive behavior of the electrode [34]. The maximum specific capacitance is $480 \mathrm{~F} \mathrm{~g}^{-1}$ at $0.2 \mathrm{~A} \mathrm{~g}^{-1}$ as demonstrated in Fig. 1g. Moreover, the PANI hydrogel electrode illustrates superior rate performance, and the capacitance remains $450 \mathrm{~F} \mathrm{~g}^{-1}$ when the current density is increased to $5 \mathrm{~A} \mathrm{~g}^{-1}$. The high rate performance may be attributed to the facile electronic and ionic transport stemming from the hydrogel materials. The cycling performance of the PANI hydrogel electrodes shows $83 \%$ retention over 10,000 cycles at $5 \mathrm{~A} \mathrm{~g}^{-1}$, which is better than that of common PANI solid electrode (typical $60 \%-85 \%$ retention for over 1000 cycles). The superior cyclic stability is proposed for the unique advantages of the cross-linking structure of PANI hydrogel that is more stable. Besides, highly porous interconnected structures in the PANI hydrogel can accommodate the swelling and shrinking of the 

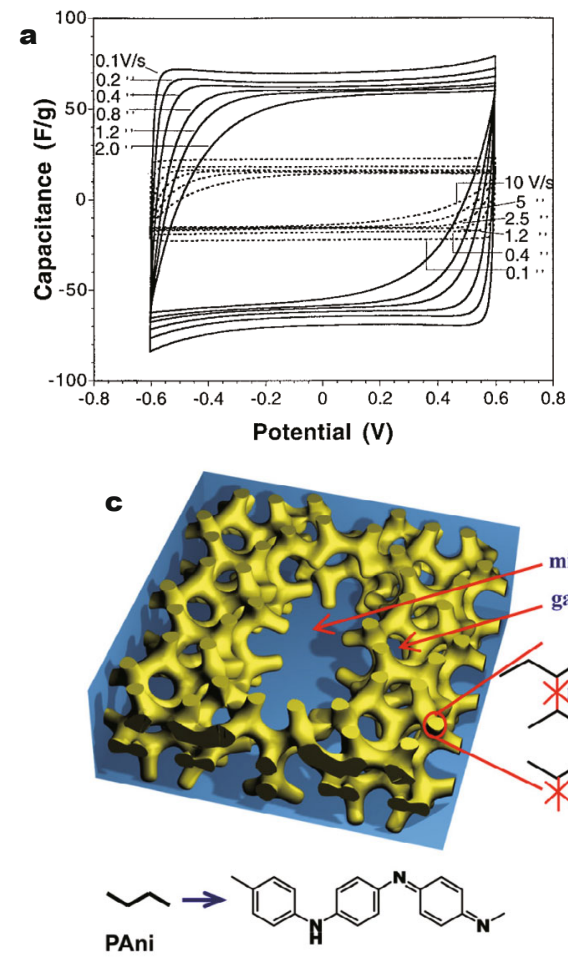

PAni

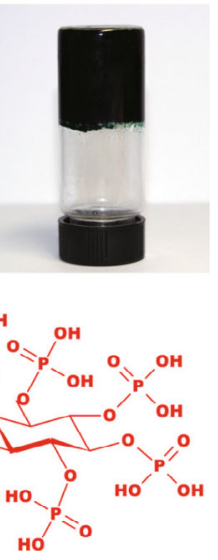

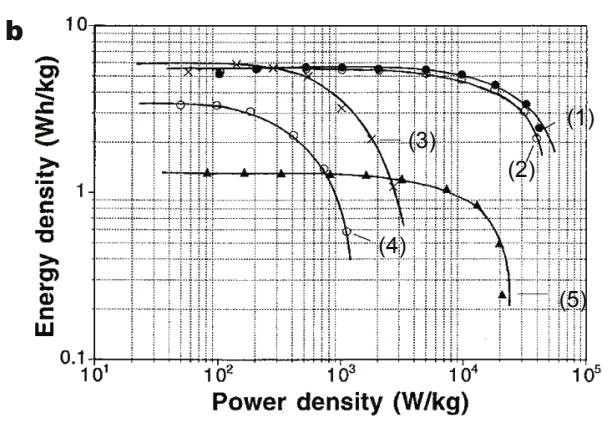
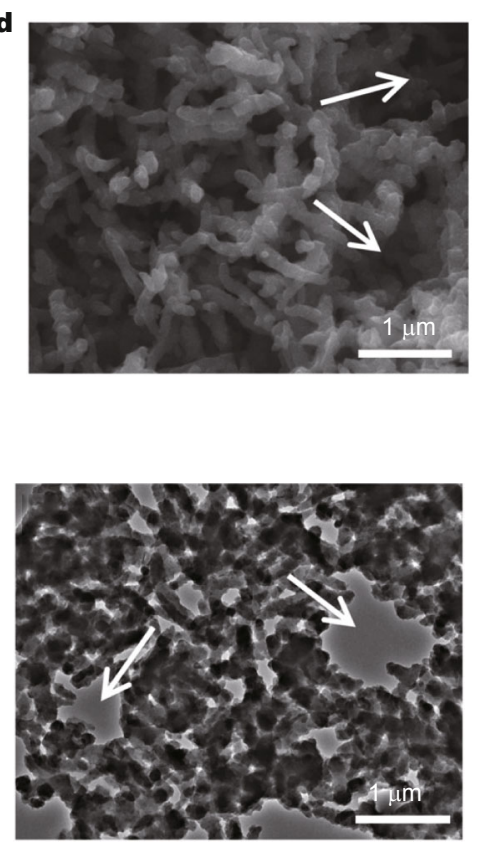

f

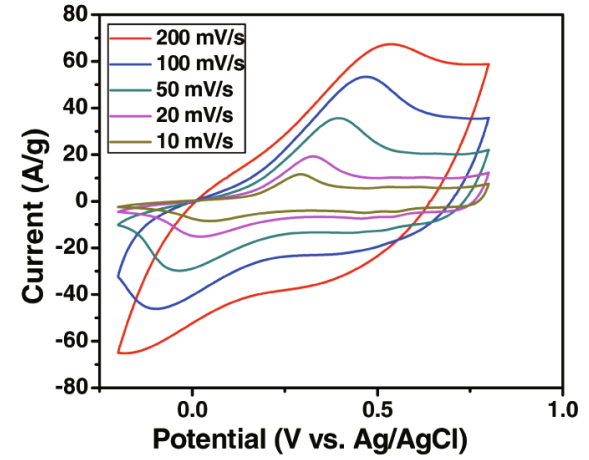

$\mathbf{g}$

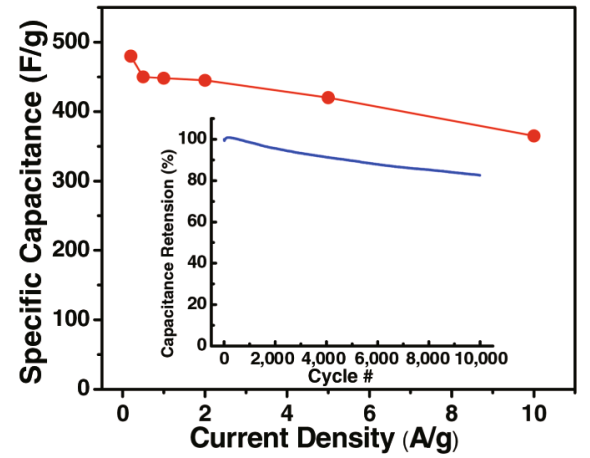

Figure 1 The preparation and electrochemical performance of pure conducting polymer hydrogel electrodes. (a) CV curves of the w-PEDOT-PSS/PPy electrodes and PEDOT-PSS electrodes. (b) Ragone plots for supercapacitors with different electrodes: (1) w-PEDOT-PSS/PPy; (2) PEDOT-PSS/PPy; (3) PPy; (4) unswelled PEDOTPSS/PPy; (5) PEDOT-PSS. (c) Schematic illustrations of the gelated PANI hydrogel where phytic acid plays the role as a dopant and a crosslinker. ( $d$ and e) SEM image and TEM image of the dehydrated PANI hydrogel nanofibers. (f) CV curves of the PANI hydrogel electrode. (g) Plot of specific capacitance for the PANI hydrogel-based electrodes. (Inset image shows the cycling performance). Reproduced with permission from Ref. [19], Copyright 1999, WILEY-VCH Verlag GmbH \& Co. KGaA, Weinheim, and Ref. [23], Copyright 2012, National Academy of Sciences.

polymer.

\section{Conducting polymer hydrogel flexible electrodes and supercapacitors}

The pristine conducting polymer hydrogels show excellent conductivity and superior electrochemical capacitive storage performance. However, the poor mechanical strength and integrity of the hydrogels limit their direct application on flexible supercapacitors.

We prepared carbon cloth supported PANI hydrogel electrode via in-situ PANI growth process, and further assembled flexible solid-state supercapacitors [35]. According to our experiments, the PANI hydrogels can be synthesized by aforementioned phytic acid cross-linking process or just employing a concentrated aniline solution without using phytic acid, in which the concentration of 
aniline monomer is no less than $0.5 \mathrm{~mol} \mathrm{~L}^{-1}$ (Fig. 2a). A carbon fiber cloth was immersed into aniline solution before adding oxidant (also known as initiator). After completing PANI polymerization and gelation, the PANI was loaded on the carbon fiber to form carbon cloth (CC) -supported PANI hydrogel electrodes with excellent flexibility and integrity. The morphologies of the pure carbon cloth and CC-supported PANI hydrogel (dehydrated PANI) are shown in Figs $2 b-d$. The electrochemical properties of the CC-supported PANI hydrogel electrodes were firstly characterized by a three-electrode setup with $1 \mathrm{~mol} \mathrm{~L}^{-1}$ aqueous
$\mathrm{H}_{2} \mathrm{SO}_{4}$. As seen in Fig. 2e, the capacitance of the electrode at $20 \mathrm{mV} \mathrm{s}^{-1}$ is around $750 \mathrm{~F} \mathrm{~g}^{-1}$, in which the loading amount of PANI hydrogel is $0.5 \mathrm{mg} \mathrm{cm}{ }^{-2}$ (dry weight). In addition, the electrode exhibits the superior rate capability at high scanning rates. The capacitance can still reach 200 $\mathrm{F} \mathrm{g}^{-1}$ even when the scanning rate increases to $200 \mathrm{mV} \mathrm{s}^{-1}$ (10 times higher than $20 \mathrm{mV} \mathrm{s}^{-1}$ ).

Furthermore, a flexible solid-state supercapacitor was fabricated based on two CC-supported PANI hydrogel electrodes and PVA- $\mathrm{H}_{2} \mathrm{SO}_{4}$ gel polymer electrolytes with the symmetric two-electrode configuration, as shown in a

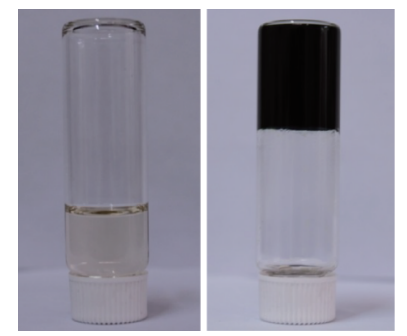

d

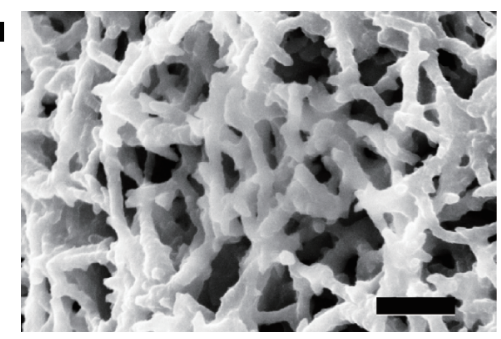

b
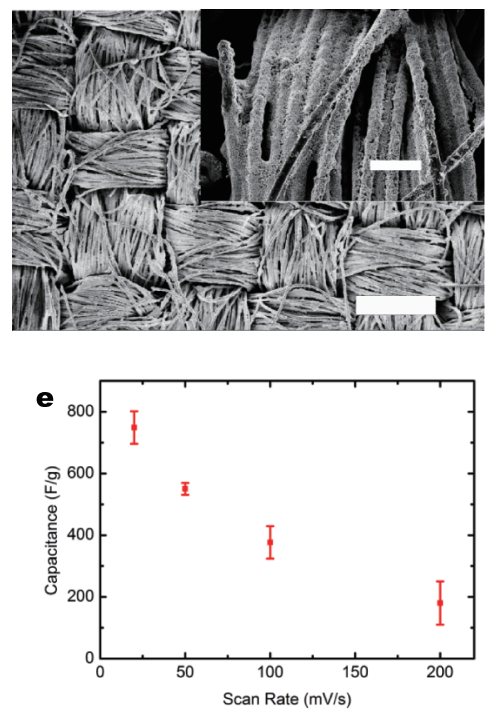

c

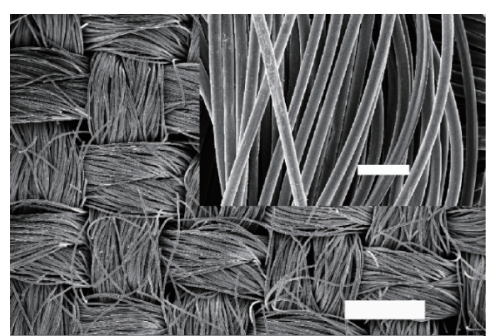

9

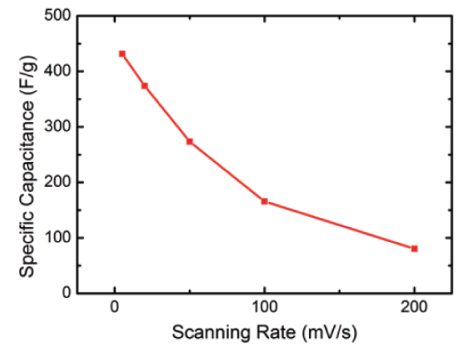

f

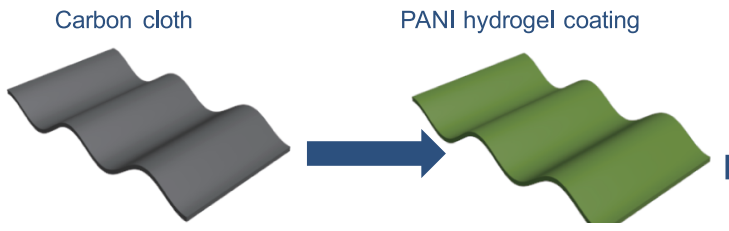

Gel electrolytes coating
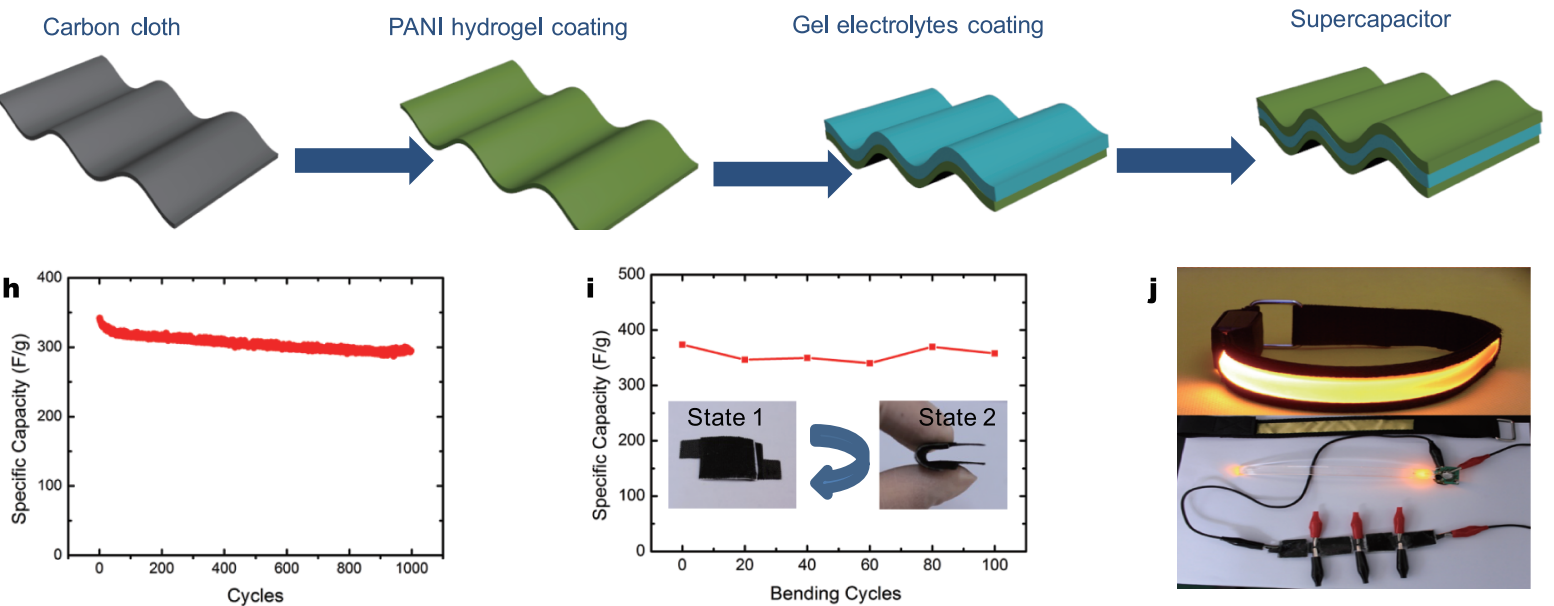

Figure 2 Conducting polymer hydrogels for flexible solid-state supercapacitors. (a) Tube inversion examination of the PANI hydrogel. (b and c) The SEM images showing the morphologies of carbon cloth and PANI hydrogel coated carbon cloth, respectively (the scale bar is $400 \mu \mathrm{m}$ ); the inset images is the enlarged SEM images (the scale bar is $40 \mu \mathrm{m}$ ). (d) The enlarged dehydrated PANI hydrogel morphologies (the scale bar is $400 \mathrm{~nm}$ ). (e) Capacitance plots with three-electrode setup. (f) Schematic of the preparation process of the PANI hydrogel-based solid-state supercapacitors. (g) Capacitance plots at varied scanning rates with two-electrode device. (h) Cycling performance at $7.5 \mathrm{~A} \mathrm{~g}^{-1}$. (i) In situ flexibility tests of the PANI hydrogel-based supercapacitors. (j) A glow armlet is driven by four as-prepared devices Supercapactiors connected in series. Reproduced with permission from Ref. [35], Copyright 2014, the Royal Society of Chemistry. 
Fig. 2f. The maximum capacitance of the supercapacitor at $5 \mathrm{mV} \mathrm{s}^{-1}$ is $430 \mathrm{~F} \mathrm{~g}^{-1}$, as calculated based on the dried weight of the PANI hydrogel (Fig. 2g). In the case of the traditional solid-state supercapacitor, the gel electrolytes hardly diffuse into the inside of the solid electrode due to the large viscosity. Therefore, the inner active materials cannot make sufficient contact with the electrolytes. However, the PANI hydrogel materials possess a $3 \mathrm{D}$ hierarchical porous structure, swelling with liquids and hence facilitating ions and mass transport, which benefits the inner active materials accessible to electrolytes and leads to a high capacitance performance. The cycling performance of the device was also studied based on galvanostatic charge-discharge measurements. After 1000 charge-discharge cycles, the capacitance can retain $86 \%$ of the original capacitance, which shows superior cycling performance compared with PANI solid electrode materials (Fig. 2h). The supercapacitor also demonstrates remarkable flexibility, as seen in Fig. 2i. The capacitance and the equivalent series resistance of the devices show no obviously deterioration even after 100 bending cycles. As a demo experiment (Fig. 2j), the hydrogel electrode-based supercapacitors were used to drive a glow armlet work that demonstrated the potential as power sources in the real application.

Besides of textile substrates, the high strength insulating hydrogel is used as substrates to load conducting polymer in order to prepare free-standing flexible hydrogel electrodes. Hao et al. [36] reported a highly flexible PANI-containing conductive hydrogel based on preorganized $\alpha$-cyclodextrins polyacrylamide ( $\alpha$-CD-PAAm) host hydrogels. Firstly, the host $a-C D$-PAAm hydrogel was prepared through a sol gel process, which contained amphiphilic domains that allowed aniline (ANI) molecules gathering in the nonpolar cavities of $\alpha$-CD. After loading ANI monomers, the in-situ polymerization can be triggered directly by immersing the hydrogel into hydrochloric acid. The resulted $\alpha$-CD- PAAm-PANI hydrogel is highly stretchable and flexible, and can withstand deformation from arbitrary direction. From scanning electron microscopy (SEM) images, the dried $\alpha$-CD-PAAm-PANI hydrogel reveals a uniform wrinkled surface texture with interconnected pores throughout the whole inner structure. The electrode was first soaked in electrolyte to reach the fully wetted state before each electrochemical test. To assemble a symmetrical two-electrode supercapacitor, two pieces of hydrogels with the same size were first attached on two $\mathrm{Pt}$ foils, which were separated by a glass fiber separator. The prototype supercapacitor was immersed in $1 \mathrm{~mol} \mathrm{~L}^{-1}$ $\mathrm{H}_{2} \mathrm{SO}_{4}$ electrolyte after it was wrapped with parafilm. The maximum specific capacitance of the hydrogel electrode in supercapacitor is $315 \mathrm{~F} \mathrm{~g}^{-1}$ on the basis of total PANI (dry weight) according to galvanostatic charge-discharge tests. Given the mass ratio (ca.13 wt.\%) of PANI in the hybrid polymer, the specific capacitance is $41 \mathrm{~F} \mathrm{~g}^{-1}$ on the basis of the whole dry mass. This a-CD-PAAm-PANI hydrogel electrode shows superior capacitance retention up to $80 \%$ after 45,000 charge-discharge cycles at $8 \mathrm{~A} \mathrm{~g}^{-1}$. In this configuration, the $\alpha$-CD-PAAm is not only a host structure but also a space buffer that could greatly accommodate the possible volume change (swelling and shrinking) of PANI during the intensive cycling processes, which results in superior cyclic stability. However, the as-prepared supercapacitor device is supported on $\mathrm{Pt}$ foil, which is not a flexible device. Besides, the device employs liquid electrolyte, which has the leakage issue especially for the flexible device with the requirement of consecutive bending cycles.

\section{Novel configuration of flexible solid-state supercapacitor with hydrogel electrodes}

A solid-state supercapacitor usually possesses a laminated configuration involving multiple interfaces, such as the multi-interfaces of cathode-electrolyte-anode. The presence of multiple interfaces facilitates the charge transport from the cathode to the anode. Nevertheless, the unavoidable relative displacement among the multilayers during bending cycles may cause delamination of these electrode and electrolyte layers, which will undoubtedly decrease the device performance. Thus, the development of new conceptual device configuration to overcome the issues of multilayer laminated device is highly required.

We developed a novel all-in-one flexible supercapacitor prototype, which integrated the main components in a single chemical hydrogel film as schemed in Fig. 3a [37]. Firstly, a chemically cross-linked PVA- $\mathrm{H}_{2} \mathrm{SO}_{4}$ hydrogel was produced by utilizing the cross-linking reaction of PVA and glutaraldehyde (GA) with the acid as a catalyst (Fig. 3b). Then, a mould-casting process (or a blade-coating process) was performed before gelatinization completing to prepare a free-standing PVA- $\mathrm{H}_{2} \mathrm{SO}_{4}$ chemical hydrogel (PCH) film as shown in Fig. 3c. The water content is approximately $90 \%$ according to the thermo-gravimetric analysis. The PCH film showes excellent elasticity, high mechanical strength and ionic conductivity. Based on the EIS results, the bulk ionic conductivity of the $\mathrm{PCH}$ film is $0.082 \mathrm{~S} \mathrm{~cm}^{-1}$, which is comparable to that of $\mathrm{H}_{2} \mathrm{SO}_{4}$ aqueous solution (Fig. 3d). Given the tensile stress-strain tests, the PCH film can sustain up to $290 \%$ stretching under a stress 
a

PVA- $\mathrm{H}_{2} \mathrm{SO}_{4}$ mixed solution
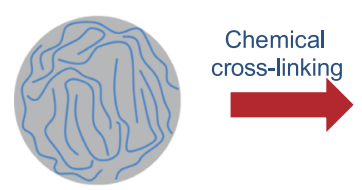

b

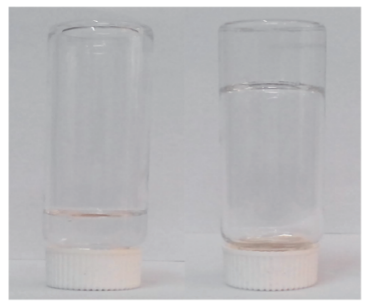

e

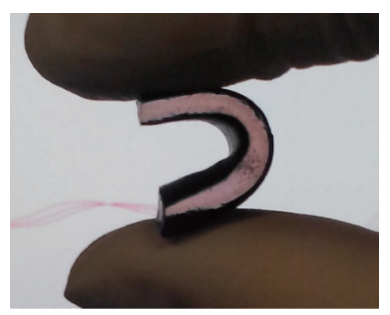

h

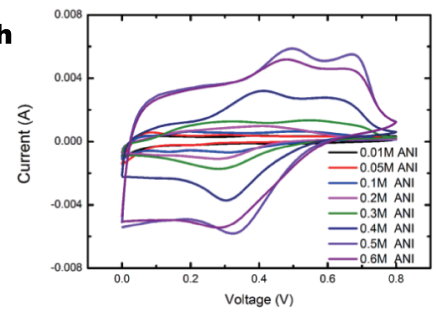

PVA- $\mathrm{H}_{2} \mathrm{SO}_{4}$ chemical hydrogel
$\mathrm{PCH}$ film
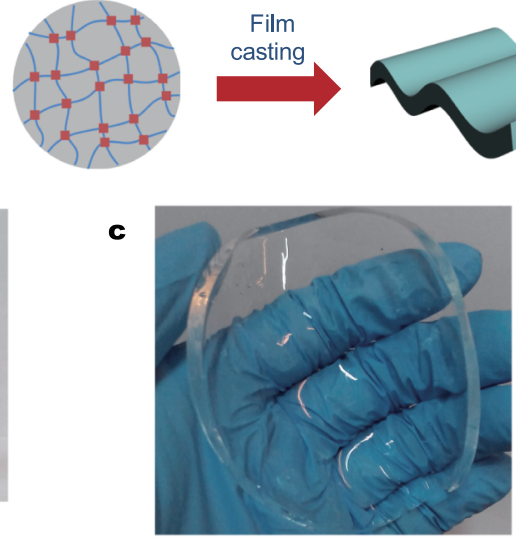

f
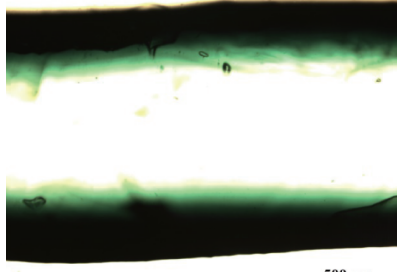

$\underline{500 \mu \mathrm{m}}$

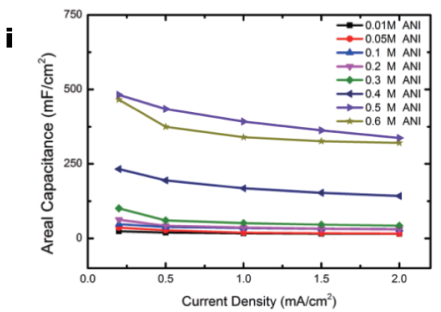

All-in-one SC based on a PANI-PCH film 
this integrated device prototype, the two PANI layers (top and down) are two electrodes, whereas the middle is the electrolyte layer. The maximum areal capacitance reaches $488 \mathrm{mF} \mathrm{cm}^{-2}$ at $0.2 \mathrm{~mA} \mathrm{~cm}^{-2}$, which is obtained based on a PANI $\left(0.5 \mathrm{~mol} \mathrm{~L}^{-1}\right)$-PCH film (Figs $\left.3 \mathrm{~h}-\mathrm{j}\right)$. To the best of our knowledge, this areal capacitance is superior to those of previous reports for two-electrode solid-state flexible supercapacitor devices [39-42]. This novel integrated configuration demonstrates a possibility to obtain high device areal capacitance via effectively improving loading amounts of active materials. Most electrode materials (PANI) were embedded inside the hydrogel film but not stacked outside the gel electrolytes, which guaranteed that the electrode materials have a sufficient contact with the gel electrolyte even under a large loading amount per area, which benefits the enhancement of capacitance. In general, the electrode shows serious decay in capacitance when the loading amount of active materials per area is too large because the pulverization and loss of the active materials during charge-discharge process. In this research, the specific capacitance is very well kept after long-term charge-discharge cycles for all the supercapacitor prototypes. For the supercapacitors based on thick PANI layer (such as that prepared in $0.5 \mathrm{~mol} \mathrm{~L}^{-1}$ ), the capacitance retention after 10,000 cycles is $99 \%$. Due to most PANI in PANI-PCH films is embedded into the PVA hydrogel, the PVA hydrogel can stabilize the PANI and bring superior cyclic stability of the device.

Moreover, the integrated supercapacitor devices show superior flexibility. The capacitance was very well maintained even after 1000 completely bending cycles. Furthermore, other commonly used conducting polymers (PPy or PEDOT, etc.) can also be used as the electrode materials to produce the prototyped supercapacitor. For example, we also prepared a PEDOT-PCH film according to the similar approach. Thus, the free-standing polymer chemical hydrogel film system is relatively versatile and robust. With this integrated configuration, the flexible solid-state supercapacitors can effectually reduce the interface transport resistance and avoid the mechanical delamination of multilayers in consecutive bending cycles. Therefore, this integrated configuration is proposed to represent the future trend of developing high-performance flexible solid-state supercapacitors.

In summary, the conducting polymer hydrogels represent a promising class of polymeric flexible electrodes for high-performance flexible supercapacitors. Conducting polymer hydrogels could offer an enlarged interface area between the electronic transporting phase (electrode) and the ionic-transporting phase (electrolyte), which benefits supercapacitor to reach more efficient electrochemical process. Furthermore, the inherent soft of conducting polymer hydrogel make hydrogel electrode materials become ideal flexible electrodes for flexible devices.

Currently, a large number of reports are available related to the synthesis of conducting polymer hydrogels and their composites. However, the preparation of high strength, high conductivity and integrated hydrogel film remains a major challenge, which is quite important for their application in flexible supercapacitors. It still requires great effort and continued innovation on exploring simple and efficient synthetic methods to prepare such high strength conducting polymer hydrogel films. Besides, the device configuration innovation of flexible supercapacitor is highly required, with the aiming of fundamentally reducing the charge transport resistance of multiple interfaces, and improving the mechanical toleration of multilayer-laminated flexible devices during consecutive bending and even stretching process. Furthermore, the combination of novel characteristics into flexible supercapacitors to prepare functional supercapacitors, such as self-healing, stretching and transparent devices, is also highly demand. By designing and preparation of novel hydrogel electrodes, these novel characteristics can be realized that will promote the development of wearable electronics in the future

Received 29 April 2016; accepted 3 June 2016;

published online 24 June 2016

1 Wang X, Lu X, Liu B, et al. Flexible energy-storage devices: design consideration and recent progress. Adv Mater, 2014, 26: 4763-4782

2 Avila AG, Hinestroza JP. Smart textiles: tough cotton. Nat Nanotech, 2008, 3: 458-459

3 Lu X, Yu M, Wang G, et al. Flexible solid-state supercapacitors: design, fabrication and applications. Energy Environ Sci, 2014, 7: 2160-2181

4 Wang $\mathrm{K}, \mathrm{Wu} \mathrm{H}$, Meng $\mathrm{Y}$, et al. Conducting polymer nanowire arrays for high performance supercapacitors. Small, 2014, 10: 14-31

5 Yu D, Qian Q, Wei L, et al. Emergence of fiber supercapacitors. Chem Soc Rev, 2015, 44: 647-662

6 Dubal DP, Kim JG, Kim Y, et al. Supercapacitors based on flexible substrates: an overview. Energy Tech, 2014, 2: 325-341

7 Wang G, Zhang L, Zhang J. A review of electrode materials for electrochemical supercapacitors. Chem Soc Rev, 2012, 41: 797-828

8 Zhu J, Yang D, Yin Z, et al. Graphene and graphene-based materials for energy storage applications. Small, 2014, 10: 3480-3498

9 Shi W, Zhu J, Sim DH, et al. Achieving high specific charge capacitances in $\mathrm{Fe}_{3} \mathrm{O}_{4} /$ reduced graphene oxide nanocomposites. J Mater Chem, 2011, 21: 3422-3427

10 Tan HT, Rui X, Yu H, et al. Aqueous-based chemical route toward ambient preparation of multicomponent core-shell nanotubes. ACS Nano, 2014, 8: 4004-4014 
11 Wang LP, Yu L, Satish R, et al. High-performance hybrid electrochemical capacitor with binder-free $\mathrm{Nb}_{2} \mathrm{O}_{5} @$ graphene. RSC Adv, 2014, 4: 37389-37394

12 Wang J, Chen $\mathrm{L}, \mathrm{Wu}$ N, et al. Uniform graphene on liquid metal by chemical vapour deposition at reduced temperature. Carbon, 2016, 96: 799-804

13 Simon P, Gogotsi Y. Materials for electrochemical capacitors. Nat Mater, 2008, 7: 845-854

14 Miao YE, Yan J, Huang Y, et al. Electrospun polymer nanofiber membrane electrodes and an electrolyte for highly flexible and foldable all-solid-state supercapacitors. RSC Adv, 2015, 5: 26189-26196

15 Fan W, Miao YE, Zhang L, et al. Porous graphene-carbon nanotube hybrid paper as a flexible nano-scaffold for polyaniline immobilization and application in all-solid-state supercapacitors. RSC Adv, 2015, 5: 31064-31073

16 Wang K, Zou W, Quan B, et al. An all-solid-state flexible microsupercapacitor on a chip. Adv Energy Mater, 2011, 1: 1068-1072

17 Wang K, Huang J, Wei Z. Conducting polyaniline nanowire arrays for high performance supercapacitors. J Phys Chem C, 2010, 114: 8062-8067

$18 \mathrm{Xu} \mathrm{J}$, Wang $\mathrm{K}, \mathrm{Zu} \mathrm{SZ}$, et al. Hierarchical nanocomposites of polyaniline nanowire arrays on graphene oxide sheets with synergistic effect for energy storage. ACS Nano, 2010, 4: 5019-5026

19 Ghosh S, Inganäs O. Conducting polymer hydrogels as 3D electrodes: applications for supercapacitors. Adv Mater, 1999, 11: 1214-1218

20 Wang Y, Shi Y, Pan L, et al. Dopant-enabled supramolecular approach for controlled synthesis of nanostructured conductive polymer hydrogels. Nano Lett, 2015, 15: 7736-7741

21 Wang K, Zhao P, Zhou X, et al. Flexible supercapacitors based on cloth-supported electrodes of conducting polymer nanowire array/SWCNT composites. J Mater Chem, 2011, 21: 16373-16378

22 Sun JY, Zhao X, Illeperuma WRK, et al. Highly stretchable and tough hydrogels. Nature, 2012, 489: 133-136

23 Pan L, Yu G, Zhai D, et al. Hierarchical nanostructured conducting polymer hydrogel with high electrochemical activity. Proc Natl Acad Sci USA, 2012, 109: 9287-9292

24 Shi Y, Pan L, Liu B, et al. Nanostructured conductive polypyrrole hydrogels as high-performance, flexible supercapacitor electrodes. J Mater Chem A, 2014, 2: 6086-6091

25 Wang Y, Yang X, Qiu L, et al. Revisiting the capacitance of polyaniline by using graphene hydrogel films as a substrate: the importance of nano-architecturing. Energy Environ Sci, 2013, 6: $477-481$

26 Macdiarmid AG, Min Y, Wiesinger JM, et al. Towards optimization of electrical and mechanical properties of polyaniline: is crosslinking between chains the key? Synthetic Metals, 1993, 55: $753-760$

27 Vikki T, Ruokolainen J, Ikkala OT, et al. Thermoreversible gels of polyaniline: viscoelastic and electrical evidence on fusible network structures. Macromolecules, 1997, 30: 4064-4072

28 Zhao Y, Liu B, Pan L, et al. 3D nanostructured conductive polymer hydrogels for high-performance electrochemical devices. Energy
Environ Sci, 2013, 6: 2856-2870

$29 \mathrm{Lu} \mathrm{Y,} \mathrm{He} \mathrm{W,} \mathrm{Cao} \mathrm{T,} \mathrm{et} \mathrm{al.} \mathrm{Elastic,} \mathrm{conductive,} \mathrm{polymeric} \mathrm{hydrogels}$ and sponges. Sci Rep, 2014, 4: 5792

30 Han Y, Shen M, Wu Y, et al. Preparation and electrochemical performances of PEDOT/sulfonic acid-functionalized graphene composite hydrogel. Synt Metal, 2013, 172: 21-27

31 Zhou H, Yao W, Li G, et al. Graphene/poly(3,4-ethylenedioxythiophene) hydrogel with excellent mechanical performance and high conductivity. Carbon, 2013, 59: 495-502

32 Naficy S, Razal JM, Spinks GM, et al. Electrically conductive, tough hydrogels with pH sensitivity. Chem Mater, 2012, 24: 3425-3433

33 Huang H, Zeng X, Li W, et al. Reinforced conducting hydrogels prepared from the in situ polymerization of aniline in an aqueous solution of sodium alginate. J Mater Chem A, 2014, 2: 16516-16522

34 Barsoukov E, Macdonald JR (eds.). Impedance Spectroscopy Theory Experiment and Applications, 2nd Edition. New Jersey: Wiley, 2005

35 Wang K, Zhang X, Li C, et al. Flexible solid-state supercapacitors based on a conducting polymer hydrogel with enhanced electrochemical performance. J Mater Chem A, 2014, 2: 19726-19732

36 Hao GP, Hippauf F, Oschatz M, et al. Stretchable and semitransparent conductive hybrid hydrogels for flexible supercapacitors. ACS Nano, 2014, 8: 7138-7146

37 Wang K, Zhang X, Li C, et al. Chemically crosslinked hydrogel film leads to integrated flexible supercapacitors with superior performance. Adv Mater, 2015, 27: 7451-7457

38 Calvert P. Hydrogels for soft machines. Adv Mater, 2009, 21: 743-756

39 El-kady MF, Strong V, Dubin S, et al. Laser scribing of high-performance and flexible graphene-based electrochemical capacitors. Science, 2012, 335: 1326-1330

40 Wang $\mathrm{K}, \mathrm{Wu} \mathrm{H}, \mathrm{Meng} \mathrm{Y}$, et al. Integrated energy storage and electrochromic function in one flexible device: an energy storage smart window. Energy Environ Sci, 2012, 5: 8384-8389

41 Kou L, Huang T, Zheng B, et al. Coaxial wet-spun yarn supercapacitors for high-energy density and safe wearable electronics. Nat Commun, 2014, 5: 3754

42 Wang K, Meng Q, Zhang Y, et al. High-performance two-ply yarn supercapacitors based on carbon nanotubes and polyaniline nanowire arrays. Adv Mater, 2013, 25: 1494-1498

Acknowledgments This work was supported by the National Natural Science Foundation of China (51403211 and 51472238), and the Innovative-Talent Program (Institute of Electrical Engineering, Chinese Academy of Sciences).

Author contributions Wang K and Ma YW conceived the structure of this manuscript, and mainly responsible for preparing the initial manuscript. Wang K, Zhang X, Sun XZ and Ma YW contributed to general discussion.

Conflict of interest The authors declare that they have no conflict of interest. 


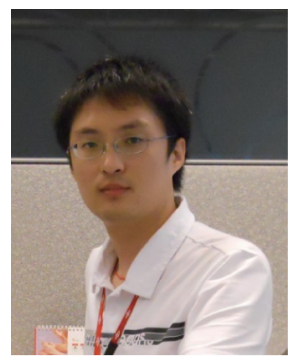

Kai Wang received his BSc degree from Shandong University (2007), and his PhD degree from the National Center for Nanoscience and Technology, Chinese Academy of Sciences under the supervision of Prof. Zhixiang Wei (2012). From 2012 to 2014, he was a postdoctoral fellow in Singapore-MIT Alliance for Research and Technology. In 2014, he joined the Institute of Electrical Engineering, Chinese Academy of Sciences as an "Innovative-Talent Program" associate professor. His research interests are in the field of electrochemical energy materials and devices, with a focus on nanomaterials, supercapacitors and integrated solid-state batteries.

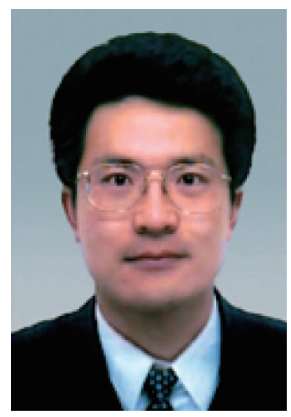

Yanwei Ma received his $\mathrm{PhD}$ degree in material processing from Tsinghua University. He had worked for six years at Tohoku University, National Institute for Materials Science, Japan and Rennes University, France, resectively. He is currently a professor and group leader at the Institute of Electrical Engineering, Chinese Academy of Sciences, Beijing. His scientific interest focused on superconducting materials and nano-materials for use in high energy supercapacitor.

\section{基于导电聚合物水凝胶材料的高性能柔性固态超级电容器}

王凯, 张熊, 孙现众, 马衍伟*

摘要 不同于固体电极, 导电聚合物水凝胶电极因含有大量水和离子可以使得导电聚合物与电解质实现分子层次的直接接触, 这会使得电 化学反应过程更加高效. 此外, 水凝胶内在的柔软特性, 会赋予电极优越的柔性特点, 有利于制备高柔性电子器件. 本文简要介绍了基于导 电聚合物水凝胶电极材料的柔性固态超级电容器的最新研究进展, 并对未来研究方向做了展望. 